\title{
Associations of progression to diabetes and regression to normal glucose tolerance with development of cardiovascular and microvascular disease among people with impaired glucose tolerance: a secondary analysis of the 30 year Da Qing Diabetes Prevention Outcome Study
}

\author{
Yanyan Chen ${ }^{1}$ (D) Ping Zhang ${ }^{2}$ (D) Jinping Wang ${ }^{3} \cdot$ Qiuhong Gong $^{1} \cdot$ Yali An $^{1} \cdot$ Xin Qian $^{1}$ (D) $\cdot$ Bo Zhang $^{4} \cdot \mathrm{Hui}^{\mathrm{Li}^{3}} \cdot$ \\ Edward W. Gregg ${ }^{5}$ - Peter H. Bennett ${ }^{6}$ (D) Guangwei $\mathrm{Li}^{1,4}$
}

Received: 23 September 2020 / Accepted: 4 December 2020 / Published online: 19 February 2021

(C) The Author(s), under exclusive licence to Springer-Verlag GmbH, DE part of Springer Nature 2021

\begin{abstract}
Aims/hypothesis We aimed to determine associations of regression to normal glucose tolerance (NGT), maintaining impaired glucose tolerance (IGT) or progression to diabetes with subsequent risks of CVD and microvascular disease among Chinese adults with IGT.

Methods We conducted an observational study among 540 participants in the Da Qing Diabetes Prevention Study, a 6 year lifestyle intervention trial in people with IGT, defined by $1985 \mathrm{WHO}$ criteria as fasting plasma glucose $<7.8 \mathrm{mmol} / \mathrm{l}$ and $2 \mathrm{~h}$ postload plasma glucose $\geq 7.8$ and $<11.1 \mathrm{mmol} / 1$. At the end of the trial, the groups that had regressed to NGT, remained with IGT or progressed to diabetes were identified. Participants were then followed for 24 years after completion of the trial, during which we compared the incidence and hazard ratios for CVD and microvascular disease in each group and estimated the differences in their median time to onset from parametric Weibull distribution models.

Results At the end of the 6 year trial, 252 (46.7\%) participants had developed diabetes, $114(21.1 \%)$ had remained with IGT and $174(32.2 \%)$ had regressed to NGT. Compared with those who developed diabetes during the trial, the median time to onset of diabetes was delayed by 14.86 years $(95 \%$ CI $12.49,17.25)$ in the NGT and 9.87 years (95\% CI 8.12 , 11.68 ) in the IGT groups. After completion of the trial, among those with diabetes, IGT and NGT, the 24 year cumulative incidence of CVD was $64.5 \%, 48.5 \%$ and $45.1 \%$, respectively, and $36.8 \%, 21.7 \%$ and $16.5 \%$ for microvascular diseases. Compared with participants who had progressed to diabetes during the trial, those who regressed to NGT had a $37 \%$ (HR $0.63 ; 95 \%$ CI $0.47,0.85)$ reduction in CVD incidence and a median delay of 7.45 years (95\% CI 1.91 , 12.99 ) in onset, and those who remained with IGT had a 34\% (HR 0.66; 95\% CI 0.47, 0.91) lower CVD incidence with a median delay in onset of 5.69 years $(95 \%$ CI 1.0, 10.38). Participants with NGT had a 66\% (HR 0.34; 95\% CI 0.20, 0.56) lower incidence of microvascular diseases and a median delay in the onset of 18.66 years (95\% CI 6.08, 31.24), and those remaining with IGT had a $52 \%$ (HR $0.48 ; 95 \%$ CI $0.29,0.81$ ) lower incidence with a median delay of 12.56 years $(95 \%$ CI $2.49,22.63)$.
\end{abstract}

Guangwei Li and Peter H. Bennett are co-senior authors and contributed equally to this article.

Guangwei Li

guangwei_li45@126.com

1 Center of Endocrinology and Cardiovascular Metabolism, Fuwai Hospital, Beijing, China

2 Division of Diabetes Translation, Centers for Disease Control and Prevention, Atlanta, GA, USA

3 Department of Cardiology, Da Qing First Hospital, Da Qing, China
4 Department of Endocrinology, China-Japan Friendship Hospital, Beijing, China

5 School of Public Health, Imperial College London, London, UK

6 Phoenix Epidemiology and Clinical Research Branch, National Institute of Diabetes and Digestive and Kidney Diseases, Phoenix, AZ, USA 


\section{Research in context}

\section{What is already known about this subject?}

- People with impaired glucose tolerance (IGT) have a high risk of progression to diabetes and cardiovascular and microvascular complications

- Lifestyle interventions can effectively prevent or delay the development of diabetes and potentially reduce the incidence of cardiovascular and microvascular complications

\section{What is the key question?}

- In individuals with IGT, are differences in response to interventions, such as progression to diabetes, regression to normal glucose tolerance (NGT) or remaining with IGT, associated with different long-term vascular outcomes?

What are the new findings?

- Participants who reverted to NGT from IGT during a 6 year lifestyle intervention trial had a $37 \%$ lower risk of cardiovascular disease events and $66 \%$ lower risk of microvascular complications during the subsequent 24 years than those who had developed diabetes at end of the 6 year trial

\section{How might this impact on clinical practice in the foreseeable future?}

- Programmes to prevent diabetes in people with IGT should be designed to prevent progression and promote regression to NGT for as long and in as many people as possible

Conclusions/interpretation People with IGT who reverted to NGT or remained with IGT at the end of the 6 year trial subsequently had significantly lower incidences of CVD and microvascular disease than those who had developed diabetes.

Keywords Cardiovascular disease - Diabetes - Impaired glucose tolerance $\cdot$ Microvascular disease $\cdot$ Normal glucose tolerance . Prevention · Progression · Regression

\author{
Abbreviations \\ DPP Diabetes Prevention Program \\ DQDPS Da Qing Diabetes Prevention Study \\ IGT Impaired glucose tolerance \\ NGT Normal glucose tolerance
}

\section{Introduction}

The global prevalence of diabetes in adults has been increasing over recent decades, with an estimated 451 million with diabetes in 2017, 281\% higher than in 2000, and with a projected increase to 693 million by 2045 [1]. In China in 2013 , the number of adults with diabetes was estimated to be 119 million ( $10.9 \%$ of the adult population), and the number with prediabetes with levels of $\mathrm{HbA}_{1 \mathrm{c}} 39-46 \mathrm{mmol} /$ $\mathrm{mol}$ (5.7-6.4\%), fasting plasma glucose 5.6-6.9 $\mathrm{mmol} / \mathrm{l}$ or $2 \mathrm{~h}$ plasma glucose $7.8-11.0 \mathrm{mmol} / \mathrm{l}$, all of whom are at high risk of developing type 2 diabetes, was approximately 388 million (35.7\% of the population) [2]. The excess incidence of macroand microvascular diseases and death among people with type 2 diabetes is well documented [3,4] and people with impaired glucose tolerance (IGT) have a high risk of progression to type 2 diabetes [5, 6], CVD and mortality [7, 8].
Several randomised clinical trials have demonstrated that lifestyle interventions in people with IGT can reduce the rate of progression to diabetes [9-12]. However, the response to lifestyle modification among people with IGT is variable, as some progress to diabetes, some regress to normal glucose tolerance (NGT) and others remain with IGT. Whether and how these different responses are associated with the subsequent long-term complications such as CVD and microvascular disease is uncertain. Interventions that prevent or delay the onset of diabetes might be expected to reduce risks of complications, yet few studies have addressed this hypothesis. The 15 year follow-up of the US Diabetes Prevention Program (DPP) provided evidence that the incidence of microvascular diseases was $21 \%$ lower in women when diabetes was prevented or delayed, but data on CVD events were not reported [13, 14]. A 13 year follow-up of the Finnish Diabetes Prevention Study, while reporting prolonged success of lifestyle intervention to prevent diabetes, showed no evidence of a reduction in CVD mortality $[15,16]$. The aim of the present study was to determine if differences in glycaemic response in people with IGT who participated in the Da Qing Diabetes Prevention Study (DQDPS) were 
associated with the subsequent risks of type 2 diabetes and cardiovascular and microvascular diseases.

\section{Methods}

Study design and participants In 1986, 576 adults aged 2574 years with IGT in Da Qing, China participated in DQDPS, a 6 year cluster randomised controlled clinical trial of lifestyle intervention, at the end of which they were informed of the trial results and subsequently received routine medical care from their usual providers. Details of the design of the trial and the primary outcomes of the subsequent 20 and 30 year follow-up studies have been reported previously $[9,17,18]$.

Participants received a $75 \mathrm{~g}$ OGTT at baseline and 2 years, 4 years and 6 years after randomisation. Subsequently, followup interviews, examinations on survivors, and reviews of medical records and death certificates were conducted 30 years after randomisation to determine the incidence of diabetes, diabetes-related complications and mortality. Study participants, and proxies who served as informants for the deceased, provided written, informed consent. Institutional review boards at the Chinese Center for Disease Control and Prevention and Fuwai Hospital approved the 30 year followup study.

Outcome definitions During the trial, diabetes, IGT and NGT status was determined using WHO 1985 criteria [19] based on $75 \mathrm{~g}$ OGTT results. Diabetes was defined as fasting plasma glucose $\geq 7.8 \mathrm{mmol} / 1$ or $2 \mathrm{~h}$ post-load plasma glucose $\geq 11.1 \mathrm{mmol} / \mathrm{l}$, IGT as fasting plasma glucose $<7.8 \mathrm{mmol} / \mathrm{l}$ and $2 \mathrm{~h}$ post-load plasma glucose $\geq 7.8$ and $<11.1 \mathrm{mmol} / 1$, and NGT as fasting plasma glucose and $2 \mathrm{~h}$ post-load plasma glucose $<7.8 \mathrm{mmol} /$ 1. During the subsequent 24 year follow-up, diabetes was defined by self-reported physician-diagnosed diabetes, evidence of increased blood or plasma glucose levels in the medical records and/or use of glucoselowering medications, and for those not known to have diabetes from OGTT results using the 1985 WHO criteria. CVD was defined as non-fatal or fatal myocardial infarction, sudden death, non-fatal or fatal stroke, or hospitalisation for heart failure. Microvascular disease was defined as a composite outcome of retinopathy, defined as a history of photocoagulation, blindness from retinal disease or proliferative retinopathy; nephropathy, defined as a history of end-stage renal disease, renal dialysis, renal transplantation or death from chronic kidney disease; and neuropathy, defined as a history of leg, ankle or foot ulceration, gangrene or amputation. Two physicians, unaware of the participant's trial assignment, independently adjudicated outcomes and disagreements were resolved by a third senior physician.
Statistical analysis The follow-up time to each outcome was calculated from the end of the trial in 1992 to the date of the first occurrence of the event, or death, or 31 December 2016, or, for those lost to follow-up, the last date of contact. Descriptive statistics were used to compare the characteristics at baseline and at the end of the trial, and changes in these measurements among groups of participants who developed type 2 diabetes, reverted to NGT or remained with IGT. Data are presented as means $( \pm \mathrm{SD})$ or counts (percentages). Comparisons among these groups were made using ANOVA tests for continuous variables and $\chi^{2}$ tests for categorical variables. Incidence rates of diabetes, CVD events and microvascular complications were expressed as the number of events divided by person-years.

The cumulative incidence rates of outcomes during the 24 year follow-up were calculated using the Kaplan-Meier method. Multivariable Cox regression models using clinically meaningful variables were used to explore determinants of progression to diabetes. Cox proportional hazards analyses were used to estimate hazard ratios for differences in complication rates among the NGT, IGT and diabetes groups. Differences in time taken to develop outcome events in the NGT, IGT and diabetes groups were estimated from parametric models with a Weibull distribution and used to interpolate differences in median survival times [20,21].

To determine if findings and conclusions would be different if the original sample had been defined using 1999 WHO criteria rather than 1985 criteria, we conducted a sensitivity analysis excluding results from the 34 original study participants who had baseline fasting plasma glucose levels of $\geq 7.0$ and $<7.8 \mathrm{mmol} / \mathrm{l}$ and who would have been designated as having diabetes by 1999 WHO criteria.

The $p$ values are two-tailed, and values $<0.05$ were considered statistically significant. All analyses were conducted using SAS version 9.4 (SAS, Cary, NC, USA).

\section{Results}

By the end of the 6 year trial, 252 (46.7\%) of the 540 participants had progressed to diabetes, 174 (32.2\%) had regressed to NGT and $114(21.1 \%)$ remained with IGT (electronic supplementary material [ESM] Fig. 1). Table 1 shows their characteristics. Participants who developed diabetes during the trial had higher baseline BMI and fasting and $2 \mathrm{~h}$ postload plasma glucose concentrations compared with those who remained with IGT or had regressed to NGT.

Predictors of progression to diabetes at the end of the 6 year trial were investigated by regression analysis using the baseline clinical characteristics of age, sex, BMI, systolic blood pressure and fasting plasma glucose as independent variables. BMI (HR 1.06; 95\% CI 1.03, 1.10) and baseline fasting plasma glucose (HR 1.72; 
Table 1 Baseline and follow-up characteristics of participants according to their glycaemic status at the end of the 6 year trial

\begin{tabular}{|c|c|c|c|}
\hline Characteristic & $\operatorname{NGT}(n=174)$ & IGT $(n=114)$ & Diabetes $(n=252)$ \\
\hline \multicolumn{4}{|l|}{1986} \\
\hline Age, years & $45.2 \pm 9.70$ & $45.3 \pm 8.9$ & $45.1 \pm 8.8$ \\
\hline Female sex, no. $(\%)$ & $70(40.2)$ & $52(45.6)$ & $120(47.6)$ \\
\hline Current smoker, no. (\%) & $73(42.0)$ & $47(41.2)$ & $99(39.3)$ \\
\hline BMI, $\mathrm{kg} / \mathrm{m}^{2}$ & $25.2 \pm 4.4$ & $25.4 \pm 3.7$ & $26.6 \pm 3.6 * *$ \\
\hline \multicolumn{4}{|l|}{$\mathrm{BP}, \mathrm{mmHg}$} \\
\hline Systolic & $131.1 \pm 24.2$ & $127.8 \pm 20.1$ & $135.7 \pm 26.6^{*}$ \\
\hline Diastolic & $87.0 \pm 15.0$ & $85.1 \pm 11.9$ & $88.5 \pm 15.1$ \\
\hline Total cholesterol, mmol/1 & $5.1 \pm 1.5$ & $4.8 \pm 1.0$ & $5.2 \pm 1.3$ \\
\hline Fasting glucose, mmol/1 & $5.5 \pm 0.7$ & $5.5 \pm 0.8$ & $5.8 \pm 0.8^{* *}$ \\
\hline $2 \mathrm{~h}$ glucose, $\mathrm{mmol} / \mathrm{l}$ & $8.8 \pm 0.8$ & $9.0 \pm 0.9$ & $9.1 \pm 0.9 * *$ \\
\hline \multicolumn{4}{|l|}{1992} \\
\hline BMI, $\mathrm{kg} / \mathrm{m}^{2}$ & $24.8 \pm 3.2$ & $25.0 \pm 3.6$ & $25.8 \pm 3.4^{*}$ \\
\hline \multicolumn{4}{|l|}{$\mathrm{BP}, \mathrm{mmHg}$} \\
\hline Systolic & $127.8 \pm 20.1$ & $133.2 \pm 19.9$ & $132.2 \pm 20.6$ \\
\hline Diastolic & $83.8 \pm 13.2$ & $85.6 \pm 11.3$ & $85.5 \pm 12.1$ \\
\hline Total cholesterol, mmol/1 & $5.1 \pm 1.4$ & $4.9 \pm 0.8$ & $5.4 \pm 1.0 *$ \\
\hline Fasting glucose, mmol/1 & $5.5 \pm 0.9$ & $5.9 \pm 0.9$ & $8.5 \pm 3.8^{* *}$ \\
\hline $2 \mathrm{~h}$ glucose, $\mathrm{mmol} / \mathrm{l}$ & $6.3 \pm 1.0$ & $9.3 \pm 0.9 * *$ & $14.3 \pm 3.5 * *$ \\
\hline \multicolumn{4}{|l|}{ Change of 6 years } \\
\hline$\Delta \mathrm{BMI}, \mathrm{kg} / \mathrm{m}^{2}$ & $-0.3 \pm 2.2$ & $-0.4 \pm 1.9$ & $-0.8 \pm 1.8^{*}$ \\
\hline \multicolumn{4}{|l|}{$\Delta \mathrm{BP}, \mathrm{mmHg}$} \\
\hline$\Delta$ Systolic & $-1.6 \pm 21.2$ & $6.3 \pm 17.8 * *$ & $-3.5 \pm 23.5$ \\
\hline$\Delta$ Diastolic & $-2.9 \pm 15.7$ & $0.7 \pm 13.2$ & $-2.7 \pm 15.2$ \\
\hline$\Delta$ Total cholesterol, $\mathrm{mmol} / \mathrm{l}$ & $0.2 \pm 1.7$ & $0.02 \pm 1.0$ & $0.1 \pm 1.6$ \\
\hline$\Delta$ Fasting glucose, $\mathrm{mmol} / \mathrm{l}$ & $0.2 \pm 1.1$ & $0.3 \pm 1.0$ & $2.7 \pm 3.7 * *$ \\
\hline$\Delta 2 \mathrm{~h}$ glucose, $\mathrm{mmol} / \mathrm{l}$ & $-2.4 \pm 1.2$ & $0.3 \pm 1.2 * *$ & $5.1 \pm 3.6^{* *}$ \\
\hline
\end{tabular}

Means \pm SD

Comparing with NGT, $* p<0.05, * * p<0.01$
$95 \%$ CI $1.40,2.13)$ were significantly and independently associated with progression to diabetes.
During the 24 year follow-up, the cumulative incidence of diabetes in participants who had reverted to NGT or retained
Fig. 1 Cumulative incidence of diabetes by glycaemic group at the end of the 6 year follow-up. The red line represents those who developed diabetes (DM), the purple line those who remained with IGT and the blue line those who reverted to NGT. The vertical black lines show the time after randomisation when the cumulative incidence of diabetes reached $50 \%$ (as indicated by the horizonal line) in the diabetes, IGT and NGT groups

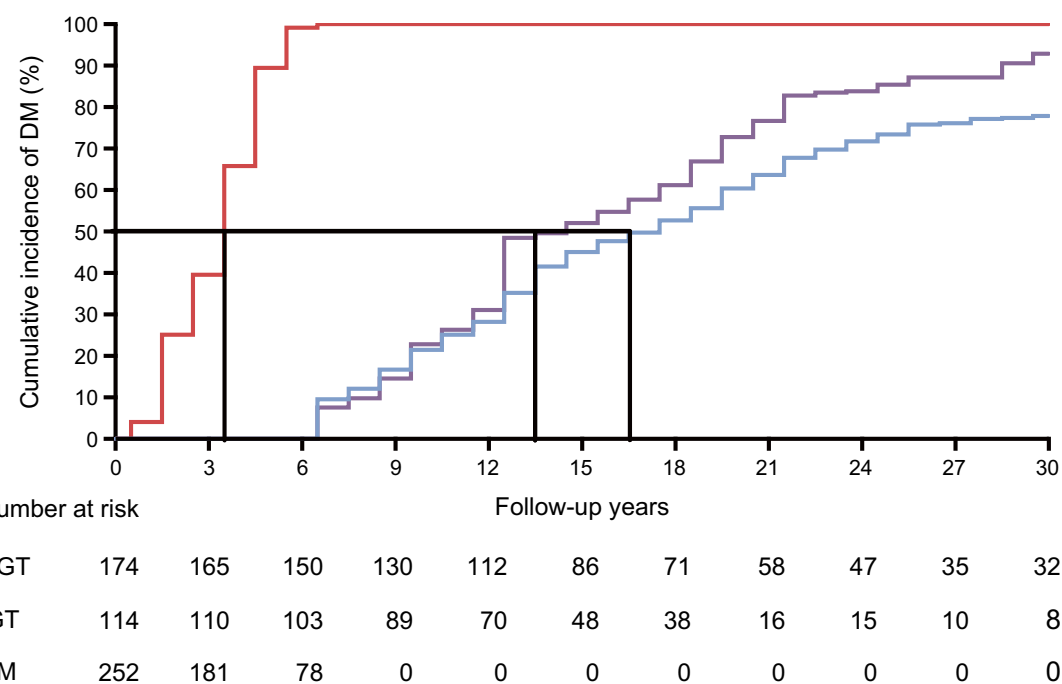


Table 2 Incidence of CVD events and microvascular disease during 24 year follow-up by glycaemic group at the end of the 6 year trial

\begin{tabular}{|c|c|c|c|c|c|c|}
\hline \multirow[t]{2}{*}{ Variable } & \multicolumn{2}{|l|}{$\begin{array}{l}\text { NGT } \\
(n=174)\end{array}$} & \multicolumn{2}{|l|}{$\begin{array}{l}\text { IGT } \\
(n=114)\end{array}$} & \multicolumn{2}{|l|}{$\begin{array}{l}\text { Diabetes } \\
(n=252)\end{array}$} \\
\hline & $\begin{array}{l}\text { Cases/person- } \\
\text { years }\end{array}$ & $\begin{array}{l}\text { Incidence } / 1000 \\
\text { person-years }(95 \% \\
\text { CI) }\end{array}$ & $\begin{array}{l}\text { Cases/person- } \\
\text { years }\end{array}$ & $\begin{array}{l}\text { Incidence } / 1000 \\
\text { person-years }(95 \% \\
\text { CI) }\end{array}$ & $\begin{array}{l}\text { Cases/person- } \\
\text { years }\end{array}$ & $\begin{array}{l}\text { Incidence } / 1000 \\
\text { person-years }(95 \% \\
\text { CI) }\end{array}$ \\
\hline CVD events ${ }^{\mathrm{a}}$ & $67 / 2672$ & $25.1(19.4,31.8)$ & $48 / 1850$ & $25.9(19.1,34.4)$ & $142 / 3629$ & $39.1(33.0,46.1)$ \\
\hline Stroke & $54 / 3674$ & $14.7(11.0,19.2)$ & $37 / 2500$ & $14.8(10.4,20.4)$ & $110 / 5221$ & $21.1(17.3,25.4)$ \\
\hline $\begin{array}{l}\text { Myocardial } \\
\text { infarction }\end{array}$ & $23 / 3025$ & $7.6(4.8,11.4)$ & $16 / 2122$ & $7.5(4.3,12.2)$ & $46 / 4508$ & $10.2(7.5,13.6)$ \\
\hline Heart failure & $12 / 3119$ & $3.8(2.0,6.7)$ & $11 / 2166$ & $5.1(2.5,9.1)$ & $32 / 4683$ & $6.8(4.7,9.6)$ \\
\hline $\begin{array}{l}\text { Composite } \\
\text { microvascular } \\
\text { disease }^{\mathrm{b}}\end{array}$ & $19 / 3062$ & $6.2(3.7,9.7)$ & $18 / 2080$ & $8.7(5.1,13.7)$ & $72 / 4215$ & $17.1(13.4,21.5)$ \\
\hline Retinopathy & $13 / 3010$ & $4.3(2.3,7.4)$ & $13 / 2033$ & $6.4(3.4,10.9)$ & $56 / 4164$ & $13.4(10.2,17.5)$ \\
\hline Nephropathy & $5 / 3129$ & $1.6(0.5,3.7)$ & $3 / 2179$ & $1.4(0.3,4.0)$ & $15 / 4687$ & $3.2(1.8,5.3)$ \\
\hline Neuropathy & $4 / 3128$ & $1.3(0.3,3.3)$ & $3 / 2173$ & $1.4(0.3,4.0)$ & $14 / 4672$ & $3.0(1.6,5.0)$ \\
\hline
\end{tabular}

${ }^{a}$ CVD events: the first occurrence of fatal and non-fatal myocardial infarction, fatal and non-fatal stroke, or hospitalised heart failure

${ }^{\mathrm{b}}$ Composite microvascular disease: the first occurrence of retinopathy, nephropathy or neuropathy

IGT increased to $77.9 \%$ (95\% CI 70.2, 83.8) and 92.9\% (95\% CI 84.0, 96.9), respectively, and in each group the cumulative incidence of diabetes reached 50\% many years later than in those who had developed it during the trial (Fig. 1).

The number of events and incidence rates of CVD and microvascular disease observed during the 24 year follow-up in the NGT, IGT and diabetes groups are shown in Table 2. The 24 year cumulative incidence of CVD was $64.5 \%$ (95\% CI $57.6,70.6)$ in the diabetes, $48.5 \%$ (95\% CI $38.2,58.1)$ in the IGT and $45.1 \%$ (95\% CI $36.8,52.9)$ in the NGT groups.

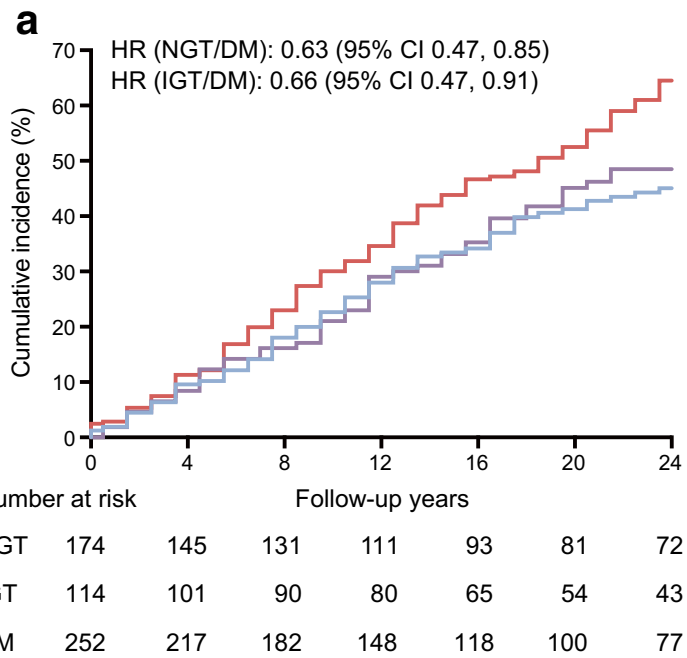

Fig. 2 Twenty-four-year cumulative incidence of first CVD events (a) and composite microvascular disease (b). The red line represents those who developed diabetes, the purple line those who remained with IGT and the blue line those who reverted to NGT by the end of the 6 year follow-up. CVD events: the first occurrence of fatal and non-fatal
CVD incidence was $37 \%$ lower (HR 0.63; 95\% CI 0.47, 0.85) in the NGT group and $34 \%$ lower (HR 0.66; $95 \%$ CI 0.47 , 0.91 ) in the IGT group than in the diabetes group (Fig. 2a, ESM Fig. 2a,b). Similar rates were also seen after adjustment for potential risk factors and in the combined NGT plus IGT group (Fig. 3, ESM Fig. 2b).

A similar pattern was seen for microvascular disease. The 24 year cumulative incidence of microvascular disease in the diabetes, IGT and NGT groups was $36.8 \%$ (95\% CI 29.9, 43.7), $21.7 \%$ (95\% CI 13.3, 31.3) and $16.5 \%$ (95\% CI 10.3,

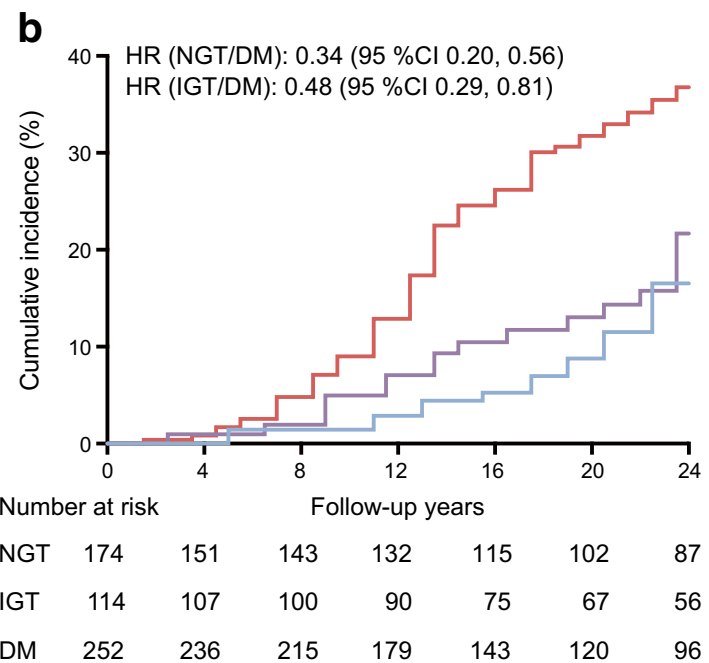

myocardial infarction, fatal and non-fatal stroke, or hospitalised heart failure. Composite microvascular disease was defined as the first occurrence of severe retinopathy, nephropathy or neuropathy. HRs are derived from proportional hazards models. DM, diabetes 


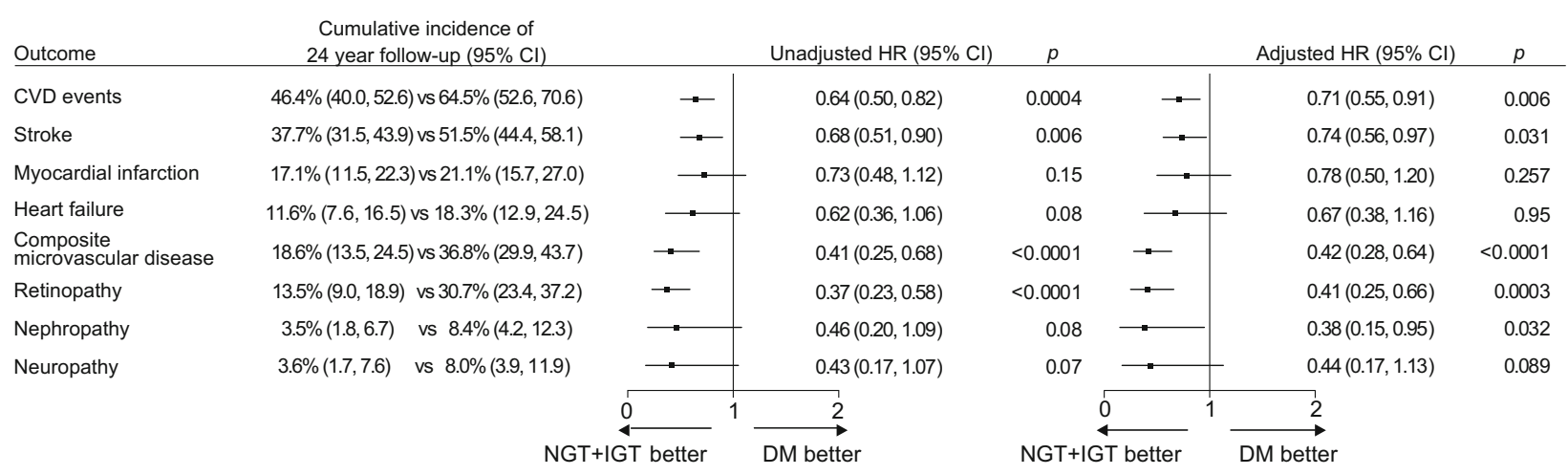

Fig. 3 Twenty-four-year cumulative incidence rates of CVD events and microvascular disease in the combined normal and impaired glucose tolerance $(\mathrm{NGT}+\mathrm{IGT})$ and diabetes $(\mathrm{DM})$ groups, with forest plots of

24.0), respectively. Incidence rates were $66 \%$ (HR 0.34; $95 \%$ CI $0.20,0.56$ ) lower in the NGT group and $52 \%$ (HR 0.48; $95 \%$ CI $0.29,0.81)$ lower in the IGT group than in the diabetes group, and remained similar and significantly lower in the NGT and IGT groups after adjustment for other risk factors (Fig. 2b, ESM Fig. 2a,b). No differences between the NGT and IGT groups were seen in the incidence of either CVD events (HR 0.94; 95\% CI 0.66, 1.40) or microvascular disease (HR $0.70 ; 95 \%$ CI $0.37,1.34$ ), nor in any of the individual components (ESM Fig. 2a).

The incidence rates of the individual components of CVD events and composite microvascular complications in the NGT, IGT and diabetes groups are shown in ESM Fig 3. The 24 year cumulative incidence rates of stroke and retinopathy were significantly lower in the NGT and IGT groups than in the diabetes group, whereas the differences in the incidence of myocardial infarction, heart failure, nephropathy and neuropathy, while lower in the NGT and IGT groups, did not reach statistical significance (ESM Fig 3a-g).

Compared with the diabetes group, the median time to onset of diabetes was 14.86 years $(95 \%$ CI $12.49,17.25)$ later in the NGT group and 9.87 years $(95 \%$ CI 8.12, 11.68) later in the IGT group. There were also longer median delays to the onset of CVD events of 7.45 years $(95 \%$ CI 1.91, 12.99) in the NGT group and 5.69 years $(95 \%$ CI 1.0, 10.38) in the IGT group. Median delays to the onset of microvascular disease of
HRs comparing the incidence of these events, unadjusted and adjusted for age, sex, smoking, BMI, systolic BP, total cholesterol and intervention

18.66 years (95\% CI 6.08, 31.24) were seen in the NGT group and of 12.56 years $(95 \%$ CI $2.49,22.63)$ in the IGT group (Table 3).

Results of the sensitivity analysis using 1999 WHO criteria rather than 1985 criteria for IGT, excluding 34 participants who otherwise would have been classified as having diabetes at baseline, are shown in the supplemental analysis (ESM Figs 4-6 and ESM Tables 1, 2). Only minor differences in the incidence and median delays in onset of CVD and microvascular disease were found.

\section{Discussion}

Most clinical trials designed to prevent type 2 diabetes have been conducted in people with IGT, who have a high risk of developing the disease $[5,6]$. The effectiveness of interventions has been judged primarily by determining the proportion who progress to glycaemic levels diagnostic for diabetes. A more clinically meaningful way to evaluate the effectiveness of such interventions would be to determine their effect on reducing the risk of diabetes-related vascular complications and mortality, which is the ultimate objective of diabetes prevention, but to do so requires many years of extended follow-up which few studies have attempted [22]. An alternative way to assess the success in the short term may be to determine the extent to

Table 3 Differences between glycaemic groups in median delay in onset of diabetes, CVD and microvascular disease

\begin{tabular}{|c|c|c|c|c|c|c|}
\hline \multirow[t]{2}{*}{ Variable } & \multicolumn{2}{|l|}{ Diabetes } & \multicolumn{2}{|l|}{$\mathrm{CVD}^{\mathrm{a}}$} & \multicolumn{2}{|l|}{ Microvascular disease $^{\mathrm{b}}$} \\
\hline & Median delay, years $(95 \% \mathrm{CI})$ & $p$ value & Median delay, years $(95 \% \mathrm{CI})$ & $p$ value & Median delay, years $(95 \% \mathrm{CI})$ & $p$ value \\
\hline NGT vs diabetes & $14.86(12.49,17.25)$ & $<0.001$ & $7.45(1.91,12.99)$ & 0.008 & $18.66(6.08,31.24)$ & 0.004 \\
\hline IGT vs diabetes & $9.87(8.12,11.68)$ & $<0.001$ & $5.69(1.0,10.38)$ & 0.017 & $12.56(2.49,22.63)$ & 0.014 \\
\hline$(\mathrm{NGT}+\mathrm{IGT})$ vs diabetes & $12.62(10.81,14.45)$ & $<0.001$ & $6.70(2.35,11.05)$ & 0.003 & $15.02(7.32,22.72)$ & $<0.001$ \\
\hline
\end{tabular}

${ }^{a} \mathrm{CVD}$ : the first occurrence of fatal and non-fatal myocardial infarction, fatal and non-fatal stroke, or hospitalised heart failure

${ }^{\mathrm{b}}$ Microvascular disease: the first occurrence of retinopathy, nephropathy or neuropathy 
which intervention results in regression to normoglycaemia or otherwise prevents progression to diabetes by prolonging the state of IGT. Whether these can be meaningful surrogate measures for the effectiveness of an intervention depends on the degree to which they ultimately predict delay in onset of diabetes and reductions in the incidence of complications, a question that we address in this paper.

It is well established that pharmacologic or lifestyle interventions in people with IGT result in diverse short-term glycaemic responses such as regression to NGT, prolongation of the period with IGT or development of diabetes [23]. However, there is only limited evidence of the effects of regression to NGT or remaining with IGT on the risk of diabetes-related vascular complications, such as CVD events and microvascular disease. For example, in the US DPP, participants with IGT who reverted to NGT during 3 years of lifestyle intervention had a $56 \%$ lower risk of type 2 diabetes during the subsequent 10 years than those who consistently had prediabetes [24] and a $22 \%$ lower risk of aggregate microvascular disease (nephropathy and retinopathy) [25], and by 15 years there was a $28 \%$ lower prevalence of microvascular complications among those who had not developed diabetes [13]. However, no difference in the incidence of CVD was seen in spite of some improvement in CVD risk factors [14]. The 15 year follow-up provided evidence that the incidence of microvascular diseases was $21 \%$ lower in women when diabetes was prevented or delayed, but data on CVD events were not reported. Similarly, a 13 year follow-up of the Finnish Diabetes Prevention Study, while reporting prolonged success of lifestyle intervention to prevent diabetes, showed no evidence of a reduction in CVD mortality rate [16].

We have previously reported that lifestyle intervention in the DQDPS reduced the incidence of diabetes, and, after 2030 years of follow-up, significantly decreased the incidence of CVD and microvascular complications and the mortality rate $[9,17,18]$. The present analysis showed that participants who remained free of diabetes during the 6 year trial subsequently had $36 \%$ lower incidence of CVD and a 59\% lower incidence of microvascular disease than those who had developed diabetes. Furthermore, the risk reductions were of similar magnitudes both in participants who regressed to NGT and in those who remained with IGT during the trial. These findings demonstrate that delaying diabetes onset in the IGT population is a key component of diabetes prevention, which ultimately results in reductions in the incidence of diabetesrelated micro- and macrovascular complications.

Compared with the participants who progressed to diabetes by the end of the 6 year trial, those who regressed to NGT or retained IGT had a much longer median delay in the onset of diabetes, and consequently experienced many fewer years of exposure to diabetes levels of hyperglycaemia and other associated metabolic abnormalities. We postulate that this decreased exposure to hyperglycaemia and the related metabolic abnormalities is the main reason for the reduced incidence of CVD and microvascular complications. Our previous reports of the effectiveness of lifestyle intervention in reducing the incidence of CVD and microvascular disease among the DQDPS participants randomised to the intervention group can be attributed largely to the greater proportion in the intervention arm $(60 \%)$ who at the end of the trial had regressed to NGT or remained with IGT vs the $36 \%$ seen in the control group [18].

The findings further emphasise that delaying diabetes onset is a key not only to curbing the increasing incidence of diabetes, but more importantly is an effective means of reducing the incidence of its serious long-term vascular complications. Participants with IGT who regressed to NGT or remained with IGT over a 6 year period had 10-15 more years free of diabetes. The findings also suggest that assessment of the proportion and duration of regression to NGT or remaining with IGT may provide useful indices to predict the likelihood of longterm success of interventions designed to prevent diabetes and its complications. Reducing the incidence of both diabetes and its complications by regressing IGT to NGT and maintaining the IGT status would result in a substantial saving in medical costs associated with treating diabetes and its complications.

The study has several strengths. First, individuals with IGT were recruited for the trial by screening a well defined population using $75 \mathrm{~g}$ OGTTs at baseline and regularly during the trial period. Second, participants were followed for up to 24 years after completing the 6 year trial, which provided enough time to observe the development of CVD events, microvascular disease and other long-term complications of diabetes. Third, little migration away from the city occurred since the start of the original trial, thereby keeping loss to follow-up to a minimum and enabling high completion rates even many years later. Lastly, all participants received usual care from the primary care clinics in Daqing, so that most participants received treatment from the same medical care system and hospital throughout the study, thereby facilitating access to their medical records. The study also has some limitations. First, the sample size was relatively small: 540 participants completed the trial and provided the clinical outcome data during follow-up. Second, we did not carry out systematic examinations at regular intervals on participants throughout the follow-up, so the outcomes assessed were limited to those that can be identified and reliably determined from medical history and other records. Third, the sample selection for the trial and this analysis are based on the 1985 WHO criteria for IGT. Supplemental analyses also indicated that the findings would have been similar if 1999 WHO criteria for IGT had been used. However, the findings may not be generalisable to people with prediabetes defined in other ways.

In conclusion, the study provides strong observational evidence that, in people with IGT, regression to NGT or otherwise prolonging the time until progression to diabetes reduces 
the risk of subsequent adverse outcomes such as CVD and microvascular complications. The findings suggest that, in people with IGT, interventions to prevent diabetes should be designed and targeted to prevent progression to diabetes and to promote regression to NGT for as long and in as many people as possible.

Supplementary Information The online version contains peer-reviewed but unedited supplementary material available at https://doi.org/10.1007/ s00125-021-05401-x.

Acknowledgements We thank all the participants in the original Da Qing Diabetes Prevention Study and the Da Qing Diabetes Prevention Outcome Study. We also thank S. Li (Medical Research and Biometrics Center, Fuwai Hospital, National Center for Cardiovascular Diseases, Peking Union Medical College, Chinese Academy of Medical Sciences, Beijing, China) and S. Liu (Department of Global Health Management and Policy, School of Public Health and Tropical Medicine, Tulane University, New Orleans, LA, USA) for their statistical assistance. We thank S. Yan (Department of Endocrinology, Harbin Fourth Hospital, Harbin, China), Y. Liu (Department of Endocrinology, 306 PLA Hospital, Beijing, China) and Y. Zhu (International Medical Department, China-Japan Friendship Hospital, Beijing, China) for their help with data collection. Special thanks are due to the late Professor Xiaoren Pan (Department of Endocrinology, China-Japan Friendship Hospital, Beijing, China); this study would not have been possible without his leadership in the original Da Qing Diabetes Prevention Study.

Data availability The data that support the findings of this study are available from the corresponding author upon reasonable request.

Funding This study was supported from 1986 to 1992 by the World Bank, the Ministry of Public Health of the People's Republic of China and Da Qing First Hospital; from 2004 to 2009 by CDC/WHO Cooperative Agreement No. U58/CCU424123-01-02, the China-Japan Friendship Hospital and Da Qing First Hospital; and from 2015 to 2018 by CDC/Chinese CDC Cooperative Agreement No. 5U19GH000636-05, the National Center of Cardiology, Fuwai Hospital, the China-Japan Friendship Hospital, Da Qing First Hospital and the Chinese Academy of Medical Sciences Innovation Fund for Medical Sciences (CIFMS) (grant number: 2020-I2M-2-006). The sponsors had no role in any aspect of the study design; in the collection, analysis and interpretation of data; or in the development of the manuscript. The findings and conclusions in this study are those of the authors and do not necessarily represent the official positions of the Centers for Disease Control and Prevention or the National Institutes of Health.

Authors' relationships and activities The authors declare that there are no relationships or activities that might bias, or be perceived to bias, their work.

Contribution statement YC designed the study, collected data, did statistical analysis and participated in drafting and writing the report. PZ coordinated and designed the study, acquired funding, did statistical analysis and contributed to writing, preparing and editing the final report. JW, QG and YA participated in the study design, acquisition and analysis of data and revising the paper. XQ participated in the acquisition of data and drafting parts of the paper. BZ and HL participated in the acquisition of data and revising the paper. EWG designed the study, acquired funding and contributed to the statistical analysis and the writing and preparation of the report. PHB designed the study, guided the data analysis and participated in writing, preparing and editing the final report. GL designed and coordinated the study, acquired funding, collected data, did statistical analysis and participated in writing and editing the final report. All authors have approved the final version of the manuscript. GL is responsible for the integrity of the work as a whole.

\section{References}

1. Cho NH, Shaw JE, Karuranga S et al (2018) IDF Diabetes Atlas: Global estimates of diabetes prevalence for 2017 and projections for 2045. Diabetes Res Clin Pract 138:271-281. https://doi.org/10. 1016/j.diabres.2018.02.023

2. Wang L, Gao P, Zhang M et al (2017) Prevalence and Ethnic Pattern of Diabetes and Prediabetes in China in 2013. JAMA 317(24):2515-2523. https://doi.org/10.1001/jama.2017.7596

3. Brownrigg JR, Hughes CO, Burleigh D et al (2016) Microvascular disease and risk of cardiovascular events among individuals with type 2 diabetes: a population-level cohort study. Lancet Diabetes Endocrinol 4(7):588-597. https://doi.org/10.1016/s2213-8587(16) 30057-2

4. Mohammedi K, Woodward M, Marre M et al (2017) Comparative effects of microvascular and macrovascular disease on the risk of major outcomes in patients with type 2 diabetes. Cardiovasc Diabetol 16(1):95. https://doi.org/10.1186/s12933-017-0574-y

5. Edelstein SL, Knowler WC, Bain RP et al (1997) Predictors of progression from impaired glucose tolerance to NIDDM: an analysis of six prospective studies. Diabetes 46(4):701-710. https://doi. org/10.2337/diab.46.4.701

6. Richter B, Hemmingsen B, Metzendorf MI, Takwoingi Y (2018) Development of type 2 diabetes mellitus in people with intermediate hyperglycaemia. Cochrane Database Syst Rev, Issue 10, Art. no.: CD012661. https://doi.org/10.1002/14651858.CD012661. pub2

7. Huang Y, Cai X, Mai W, Li M, Hu Y (2016) Association between prediabetes and risk of cardiovascular disease and all cause mortality: systematic review and meta-analysis. BMJ 355:i5953. https:// doi.org/10.1136/bmj.i5953

8. Gong Q, Zhang P, Wang J et al (2016) Changes in Mortality in People With IGT Before and After the Onset of Diabetes During the 23-Year Follow-up of the Da Qing Diabetes Prevention Study. Diabetes Care 39(9):1550-1555. https://doi.org/10.2337/dc160429

9. Pan XR, Li GW, Hu YH et al (1997) Effects of diet and exercise in preventing NIDDM in people with impaired glucose tolerance. The Da Qing IGT and Diabetes Study. Diabetes Care 20(4):537-544. https://doi.org/10.2337/diacare.20.4.537

10. Tuomilehto J, Lindstrom J, Eriksson JG et al (2001) Prevention of type 2 diabetes mellitus by changes in lifestyle among subjects with impaired glucose tolerance. N Engl J Med 344(18):1343-1350. https://doi.org/10.1056/NEJM200105033441801

11. Knowler WC, Barrett-Connor E, Fowler SE et al (2002) Reduction in the incidence of type 2 diabetes with lifestyle intervention or metformin. N Engl J Med 346(6):393-403. https://doi.org/10. 1056/NEJMoa012512

12. Ramachandran A, Snehalatha C, Mary S, Mukesh B, Bhaskar AD, Vijay V (2006) The Indian Diabetes Prevention Programme shows that lifestyle modification and metformin prevent type 2 diabetes in Asian Indian subjects with impaired glucose tolerance (IDPP-1). Diabetologia 49(2):289-297. https://doi.org/10.1007/s00125-0050097-z

13. Diabetes Prevention Program Research Group (2015) Long-term effects of lifestyle intervention or metformin on diabetes development and microvascular complications over 15-year follow-up: the Diabetes Prevention Program Outcomes Study. Lancet Diabetes 
Endocrinol 3(11):866-875. https://doi.org/10.1016/S22138587(15)00291-0

14. Perreault L, Temprosa M, Mather KJ et al (2014) Regression from prediabetes to normal glucose regulation is associated with reduction in cardiovascular risk: results from the Diabetes Prevention Program outcomes study. Diabetes Care 37(9):2622-2631. https:// doi.org/10.2337/dc14-0656

15. Lindström J, Peltonen M, Eriksson JG et al (2013) Improved lifestyle and decreased diabetes risk over 13 years: long-term follow-up of the randomised Finnish Diabetes Prevention Study (DPS). Diabetologia 56(2):284-293. https://doi.org/10.1007/s00125-0122752-5

16. Uusitupa M, Peltonen M, Lindström J et al (2009) Ten-year mortality and cardiovascular morbidity in the Finnish Diabetes Prevention Study-secondary analysis of the randomized trial. PLoS One 4(5): e5656. https://doi.org/10.1371/journal.pone.0005656

17. Li G, Zhang P, Wang J et al (2008) The long-term effect of lifestyle interventions to prevent diabetes in the China Da Qing Diabetes Prevention Study: a 20-year follow-up study. Lancet 371(9626): 1783-1789. https://doi.org/10.1016/s0140-6736(08)60766-7

18. Gong Q, Zhang P, Wang J et al (2019) Morbidity and mortality after lifestyle intervention for people with impaired glucose tolerance: 30-year results of the Da Qing Diabetes Prevention Outcome Study. Lancet Diabetes Endocrinol 7(6):452-461. https://doi.org/ 10.1016/s2213-8587(19)30093-2

19. World Health Organization (1985) Diabetes mellitus: report of a WHO Study Group. World Health Organ Tech Rep Ser 727:1-113
20. Lee ET, Go OT (1997) Survival analysis in public health research. Annu Rev Public Health 18:105-134. https://doi.org/10.1146/ annurev.publhealth.18.1.105

21. Cox C, Matheson M (2014) A comparison of the generalized gamma and exponentiated Weibull distributions. Stat Med 33(21):3772-3780. https://doi.org/10.1002/sim.6159

22. Nathan DM, Bennett PH, Crandall JP et al (2019) Does diabetes prevention translate into reduced long-term vascular complications of diabetes? Diabetologia 62(8):1319-1328. https://doi.org/10. 1007/s00125-019-4928-8

23. Cefalu WT, Buse JB, Tuomilehto J et al (2016) Update and Next Steps for Real-World Translation of Interventions for Type 2 Diabetes Prevention: Reflections From a Diabetes Care Editors' Expert Forum. Diabetes Care 39(7):1186-1201. https://doi.org/10. 2337/dc16-0873

24. Perreault L, Pan Q, Mather KJ, Watson KE, Hamman RF, Kahn SE (2012) Effect of regression from prediabetes to normal glucose regulation on long-term reduction in diabetes risk: results from the Diabetes Prevention Program Outcomes Study. Lancet 379(9833): 2243-2251. https://doi.org/10.1016/s0140-6736(12)60525-x

25. Perreault L, Pan Q, Schroeder EB et al (2019) Regression From Prediabetes to Normal Glucose Regulation and Prevalence of Microvascular Disease in the Diabetes Prevention Program Outcomes Study (DPPOS). Diabetes Care 42(9):1809-1815. https://doi.org/10.2337/dc19-0244

Publisher's note Springer Nature remains neutral with regard to jurisdictional claims in published maps and institutional affiliations. 